\title{
Recognition and Classification of Power Quality Disturbances on the basis of Pattern Linguistic Values
}

\author{
XiaoSheng Liu ${ }^{\dagger}$, Bo Liu* and DianGuo Xu*
}

\begin{abstract}
This paper presents a new recognition and classification method for power quality (PQ) disturbances on the basis of pattern linguistic values. This method solves the difficulty of recognizing disturbances rapidly and accurately by using fuzzy logic. This method uses classification disturbance patterns to define the linguistic values of fuzzy input variables and used the input variables of corresponding disturbance pattern to set membership functions. This method also sets the fuzzy rules by analyzing the distribution regularities of the input variable values. One characteristic of this method is that the linguistic values of fuzzy input variables and the setting of membership functions are not only related to the input variables but also to the character of classification disturbance and the classification results. Furthermore, the number of fuzzy rules is equal to the number of disturbance patterns. By using this method for disturbance classification, the membership function and design of fuzzy rules are directly related to the objective of classification, thus effectively reducing the complexity of the design process and yielding accurate classification results. The classification results of the simulation and measured data verify the feasibility and effectiveness of this method.
\end{abstract}

Keywords: Power quality, Disturbances classification, Fuzzy logic, S-transform

\section{Introduction}

PQ disturbances are increasing in severity in modern power networks because of the increasing use of power electronic devices, particularly for various nonlinearity, impact, and volatility loads. With the continual expansion of power networks and continuous improvement of requirements for $\mathrm{PQ}$, research attention has focused on how to improve PQ to ensure power supply reliability. Hence, the classification of PQ disturbances is the necessary premise to evaluate and improve PQ.

To realize the recognition and classification of PQ disturbances, the original waveform of disturbance signals should be transformed and reconstructed and effective features should be extracted. Common methods include short-time Fourier transform (STFT) [1], wavelet transform (WT) [2], S-transform (ST) [3], and sparse signal decomposition (SSD) [4]. Nevertheless, STFT has a single time-frequency resolution and does not satisfy the requirement that various disturbance patterns have different time-frequency resolutions in different frequency domains. Seemly, how to select the mother wavelet and the decomposition levels properly in WT is complex for obtaining a high accuracy and strong robustness. ST is the extension of continuous WT theory and can keep the absolute phase of every frequency when analyzing various resolutions. Recently, the sparse signal decomposition

$\dagger$ Corresponding Author: Dept. of Electrical Engineering, Harbin Institute of Technology, Harbin, China. (liuxsh@hit.edu.cn)

* Dept. of Electrical Engineering, Harbin Institute of Technology, Harbin, China. (liubo973@163.com,xudiang@hit.edu.cn) Received: April 30, 2015; Accepted: November 9, 2015
(SSD) technique with overcomplete hybrid dictionaries (OHDs) presents a new tool for signal expansion. However, a pending problem is how to construct the appropriate OHDs for the best approximation of certain signal patterns in the disturbances classification. Hence, the relevant features used to recognize the disturbances can be extracted simply and effectively by ST.

After obtaining the features that represent the essential information of disturbance signals, methods such as support vector machine (SVM) [5-7], binary feature matrix [8], and hidden Markov model (HMM) [9] should be used to confirm the disturbance patterns of the signal samples. These methods have successfully realized the automatic recognition of PQ disturbances. However, because of the sensitivity of noise and insufficient data, the accuracy of disturbance patterns may be affected. Given the complexity and various types of PQ disturbance signals, the application of artificial neural network (ANN) [10-13] and fuzzy logic [14-22] has gotten increasing attention. ANN, which has strong adaptivity and fault tolerance, is a powerful tool for recognizing PQ disturbances and has already obtained remarkable research results. ANN and fuzzy logic are based on probabilistic neural network (PNN) in [10] and radial basis function neural network in [11], respectively, and realize high accurate classification. However, these neural network classifiers need substantial sample data and continuous training and studying. At the same time, controlling network size and avoiding the curse of dimensionality need to be taken seriously as considerations when using these types of methods.

Similar to ANN classification, fuzzy classification is 
also liable for expressing the nonlinearity information of disturbances with strong robustness. The difference is that fuzzy logic is the stimulation of human fuzzy thinking, whereas fuzzy classification is easy to merge and use with the related experience knowledge of humans. Moreover, the algorithm complexity is relatively low compared with the ANN classification. Therefore, fuzzy classification becomes another effective method of disturbance classification. A classifier of PQ disturbances based on a rule base using DT-fuzzy (DT-F) is proposed in [15], but the complexity of the rule base of DT-F may limit its applications. The classification of disturbances in [22] uses 5 features extracted by ST, and the simplified rule base is designed with 12 rules to reduce system complexity such as additional references. To ensure the effectiveness of simplified rules, fuzzy rules must closely relate to the other parts of the fuzzy reasoning process, particularly to the fuzzy set numbers of input fuzzy variables and the shape and position parameters of membership function. However, the design of these parameters and rules is actually independent in traditional fuzzy reasoning methods.

Although every section of a fuzzy classification system is connected with each other and has a significant impact on disturbance classification results together, the classification system design process of each section still does not reflects the internal relations with each other among the existing methods. For example, the design process of linguistic value numbers and membership function parameters only relates to the values of input variables but not to the classification purpose. Many uncertain factors are present in the design process of fuzzy classification system, thus increasing the complexity of the design process and decreasing the classification accuracy. This paper attempts to improve the fuzzy reasoning approach and simplify the design process, which are suitable for solving the problems of PQ disturbances classification. Especially, the pattern of disturbances is defined as the linguistic values in the universe of input fuzzy variables; the corresponding membership function is obtained from the characteristics of input variables.

The paper is organized as follows. Section 2 explains the principle and design of the proposed approach. Sections 3 and 4 show the feature extraction by using ST and the classification process based on the proposed method, respectively. Section 5 shows the performance of the classifier for simulation and measured data. Finally, Section 6 concludes.

\section{Classification Approach based on Pattern Linguistic Values}

In fuzzy logic, the results of fuzzy reasoning are affected by several reasons, e.g., the selection of input and output variables, setting of linguistic values, definition of membership function, design of fuzzy inference, and defuzzification approach. The membership function is the bridge between fuzzy logic and accurate data and has a great influence on the final results of fuzzy reasoning as the most important calculation step.

The relationship between the membership function and value of fuzzy variables (also known as linguistic value) is always one-to-one, i.e., each value of the fuzzy variable corresponds to the membership function defined in the universe. In the traditional fuzzy logic approach, the value of fuzzy variables is related to its specific input values (shown as positive big, positive small, zero, negative small, and negative big in general). Furthermore, positive middle and negative middle may be used for fine universe dividing. On the basis of the aforementioned definition of the membership function, the number of fuzzy reasoning rules directly depends on the number of fuzzy sets of input fuzzy variables (also known as number of membership function). For example, in the fuzzy reasoning system with two inputs and a single output, if each input variable has 5 fuzzy sets, the whole fuzzy rules will be $5 * 5=25$, which is equal to an interval number in the universe.

For the fuzzy inference system to classify PQ disturbances, the input variables are always more than two generally. Therefore, the whole fuzzy rules base will be complex and large. Considering the online real-time possibility and necessity, designed logic rules are needed to make a choice in the design of fuzzy classification system of $P Q$ disturbances such that the system response speed is improved by reducing the rule number and amount of calculation. However, the choice principle is always needed to compromise and will be in a dilemma. Retaining too many rules lead to an increase in computational complexity and possible overfitting, whereas simplifying some rules lead to reduced correct rate.

The mentioned design of the linguistic value and membership function is only related to the characteristic of input fuzzy variables. Thus, this approach is suitable for conventional fuzzy reasoning system with accurate output value to act on the controlled object. However, this approach is certainly not always the best approach for the classification system because it is the fuzzy reasoning system for classifying input variables that is used to obtain discrete classification results. Moreover, this approach does not continuously provide an accurate output value. On the basis of the above situation, if the classification requirements of the reasoning system output are considered, a new design approach of fuzzy

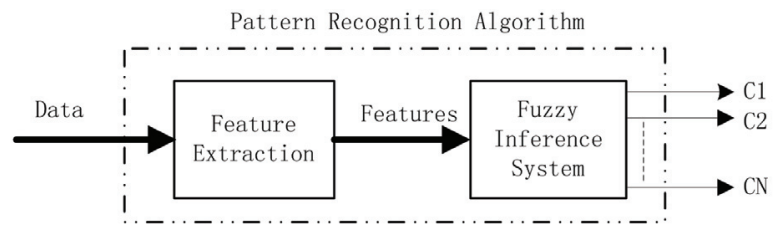

Fig. 1. Fuzzy pattern classifier 
reasoning system may be formed for the pattern classification application field.

Most works present a two-stage method to overcome the PQ fuzzy classification problems, as shown in Fig. 1, where $\mathrm{C} 1, \mathrm{C} 2, \ldots, \mathrm{CN}$ are identified as the PQ disturbance patterns. In the first stage, the time series data of disturbances is transformed into certain unique features by suitable mathematical transformation. In the second stage, the input variables of the fuzzy inference system are the aforementioned features and the output variables are classification results, which are the pattern of disturbances such as sag, swell, transients, notch, and harmonics.

On the basis of the difference between the requirements of simplifying rules and the traditional fuzzy reasoning mechanism, a new fuzzy reasoning approach is proposed: in the universe of input fuzzy variables, the pattern of disturbances (e.g., sag, swell, etc.) is directly seen as linguistic values and defines the corresponding membership function according to the characteristics of input variables. On the basis of the aforementioned, the input variables are the features reflecting the disturbance characteristics of the PQ waveform. For each signal pattern, every feature will have a corresponding range of values that need to be covered with the membership function, which is necessary with an appropriate function expression, thus indicating the suitable generalization ability.

The number of fuzzy rules is observably decreased and equal to the pattern number because of the benefit from the one-to-one correspondence between input fuzzy variable values and output classification results. In this paper, the proposed approach is called the fuzzy classification based on pattern linguistic values.

\section{Feature Extraction}

To distinguish and classify the different PQ disturbances occurring in power systems, the original signals should be processed by a time-frequency transform tool such as STFT, WT, and ST to extract relevant information about the signal. Certain unique features are extracted from the processed signal for a given PQ disturbance that may be further used for the automatic classification of the disturbance.

$\mathrm{ST}$ is a reversible local time-frequency transform tool that is viewed as a frequency-dependent STFT or a continuous WT. Given the frequency-dependent Gaussian window used for the analysis of a signal data, the multiresolution ST has a rational distribution of frequency resolution and can be independent of each frequency component of the signal amplitude variation. Therefore, the independent multi-resolution ST has been proven to perform effectively.

The multi-resolution ST of a signal $h(t)$ is defined as follows:

$$
\begin{gathered}
S(\tau, f)=\int_{-\infty}^{\infty} h(t) g(\tau-t, f) e^{-i 2 \pi f t} \mathrm{~d} t \\
g(\tau, f)=\frac{|f|}{k \sqrt{2 \pi}} e^{-\frac{\mathrm{t}^{2} f^{2}}{2 k^{2}}}
\end{gathered}
$$

where the parameter $k$ sets the width of the Gaussian window for a given frequency. For $k>1$, the frequency resolution improves and the time resolution deteriorates. The reverse occurs with $k<1$.

The PQ signal $h(t)$ can be expressed in discrete form as $h(K T), \quad k=0,1,2, \ldots N-1$, with $\mathrm{T}$ as the sampling time interval. By using (1), the ST of a discrete time series $h(K T)$ is obtained by expressing $f \rightarrow n / N T$ and $\tau \rightarrow n / j T$ as

\begin{tabular}{|c|c|c|c|}
\hline Pattern of signals & Class symbol & Equation & Parameters \\
\hline Normal & $\mathrm{C} 1$ & $v(t)=A \cos (\omega t)$ & $A=1(\mathrm{pu}), f=50 \mathrm{~Hz}, \omega=2 \pi f$ \\
\hline Sag & $\mathrm{C} 2$ & $v(t)=A\left\{1-k\left[u\left(t_{2}\right)-u\left(t_{1}\right)\right]\right\} \cos (\omega t)$ & $0.1 \leq k \leq 0.9 ; T \leq t_{2}-t_{1} \leq 9 T$ \\
\hline Swell & $\mathrm{C} 3$ & $v(t)=A\left\{1+k\left[u\left(t_{2}\right)-u\left(t_{1}\right)\right]\right\} \cos (\omega t)$ & $0.1 \leq k \leq 0.8 ; T \leq t_{2}-t_{1} \leq 9 T$ \\
\hline Interruption & $\mathrm{C} 4$ & $v(t)=A\left\{1-k\left[u\left(t_{2}\right)-u\left(t_{1}\right)\right]\right\} \cos (\omega t)$ & $0.9 \leq k \leq 1 ; T \leq t_{2}-t_{1} \leq 9 T$ \\
\hline Harmonics & $\mathrm{C} 5$ & $v(t)=A \cos (\omega t)+h_{3} \cos (3 \omega t)+h_{5} \cos (5 \omega t)+h_{7} \cos (7 \omega t)$ & $0.05 \leq h_{i} \leq 0.25, i=3,5,7$ \\
\hline Flicker & C6 & $v(t)=A[1+\alpha \cos (\beta \omega t)] \cos (\omega t)$ & $0.1 \leq \alpha \leq 0.2 ; 0.1 \leq \beta \leq 0.5$ \\
\hline Transient & $\mathrm{C} 7$ & $\begin{aligned} v(t)=A[ & \cos (\omega t)+k \exp \left(-\left(t-t_{1}\right) / \tau\right) \cos \left(\omega_{n}\left(t-t_{1}\right)\right) \\
& \left.\times\left(u\left(t_{2}\right)-u\left(t_{1}\right)\right)\right]\end{aligned}$ & $\begin{array}{c}0.1 \leq k \leq 0.8 ; \tau=0.0015 \\
100 \mathrm{~Hz} \leq \omega_{n} \leq 1300 \mathrm{~Hz} ; \quad T \leq t_{2}-t_{1} \leq 9 T\end{array}$ \\
\hline Spike & $\mathrm{C} 8$ & $v(t)=\sin \omega t+\operatorname{sgn}(\sin \omega t) A\left[u\left(t-t_{1}\right)-u\left(t-t_{2}\right)\right]$ & $\begin{array}{c}t_{1}=0.0048+0.01 i, \quad t_{2}=0.0052+0.01 i \\
i=0,1, \cdots, \quad 0.5 \leq A \leq 1.5\end{array}$ \\
\hline Notch & C9 & $v(t)=\sin \omega t-\operatorname{sgn}(\sin \omega t) A\left[u\left(t-t_{1}\right)-u\left(t-t_{2}\right)\right]$ & $\begin{array}{c}t_{1}=0.0045+0.01 i, \quad t_{2}=0.0055+0.01 i \\
i=0,1, \cdots, \quad 0.1 \leq A \leq 0.4\end{array}$ \\
\hline Sag+harmonic & $\mathrm{C} 10$ & $\begin{aligned} v(t)= & A\left\{1-k\left[u\left(t_{2}\right)-u\left(t_{1}\right)\right]\right\} \\
& \left(\cos (\omega t)+h_{3} \cos (3 \omega t)+h_{5} \cos (5 \omega t)+h_{7} \cos (7 \omega t)\right)\end{aligned}$ & $\begin{array}{c}0.1 \leq k \leq 0.9 ; T \leq t_{2}-t_{1} \leq 9 T \\
0.05 \leq h_{i} \leq 0.25, \quad i=3,5,7\end{array}$ \\
\hline Swell+harmonic & $\mathrm{C} 11$ & $\begin{aligned} v(t)= & A\left\{1+k\left[u\left(t_{2}\right)-u\left(t_{1}\right)\right]\right\} \\
& \left(\cos (\omega t)+h_{3} \cos (3 \omega t)+h_{5} \cos (5 \omega t)+h_{7} \cos (7 \omega t)\right)\end{aligned}$ & $\begin{array}{c}0.1 \leq k \leq 0.8 ; T \leq t_{2}-t_{1} \leq 9 T \\
0.05 \leq h_{i} \leq 0.15, \quad i=3,5,7\end{array}$ \\
\hline
\end{tabular}

Table 1. PQ disturbance models 
follows:

$$
\left\{\begin{array}{l}
S\left[j T, \frac{n}{N T}\right]=\sum_{m=0}^{N-1} H\left[\frac{m+n}{N T}\right] e^{-\frac{2 \pi^{2} 2_{m} 2}{n^{2}} e^{\frac{j 2 \pi i m}{N}}, \quad n \neq 0} \\
S[j T, 0]=\frac{1}{N} \sum_{m=0}^{N-1} h\left[\frac{m}{N T}\right], \quad n=0
\end{array}\right.
$$

where $j, m$, and $n=0,1, \ldots N-1$.

The fuzzy classifier on the basis of pattern linguistic value presented in this work is designed to recognize 11 disturbances. Table 1 provides a detailed summary of all disturbances of patterns with the equations and controlling parameters $[24,25]$. A total of 100 cases of each pattern with different parameters were generated and utilized by MATLAB 7.10.0. Another 100 cases of each pattern that are different from the training set were generated randomly for testing the proposed algorithm. The sampling frequency of the data set is $3.2 \mathrm{kHz}$, which is commonly used in practical applications, whereas the data length of each class of signals is 16 cycles. Certain unique features for all types of PQ disturbances are extracted from the S-matrix.

Figs. 2 and 3 show the ST outputs for the disturbance signals of the voltage sag and transient, including the signal waveform, time-frequency contours (also known as S-matrix contours), amplitude, and frequency contents of the signals. For simplicity, only two types of 11 disturbance signals are shown here.

The S-matrix contours provide the information regarding the time localization of the disturbance data. As shown in the figures, time localization occurs in the frequency
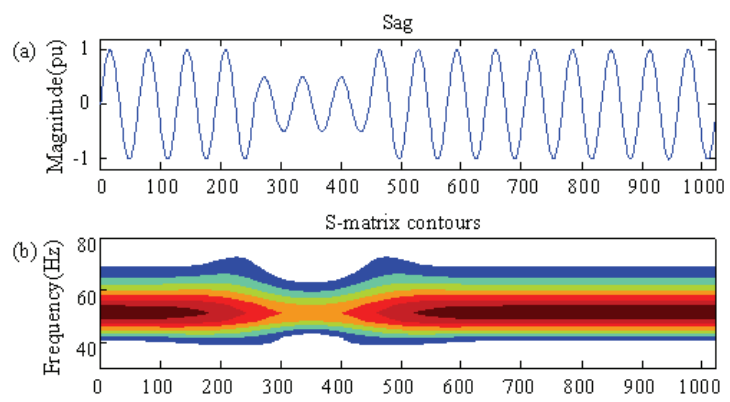

Amplitude vs time
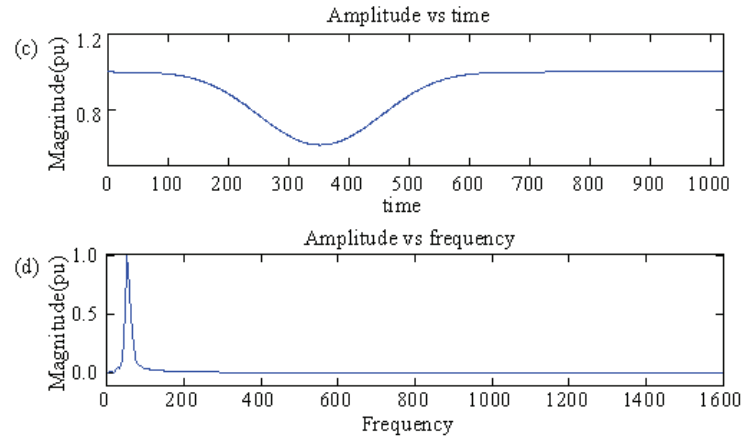

Fig. 2. (a) Voltage sag waveform; (b) S-matrix contours of sag; (c) Magnitude-time curve from S-matrix; (d) Amplitude-frequency curve from S-matrix domain instantly with the disturbance in the time scale. Both the amplitude and frequency variations of the disturbance signal are also calculated from the S-matrix contours. By comparing Fig. 2(d) shows that only one peak pertaining to the fundamental frequency appears, whereas Fig. 3(d) shows that two peaks characterize the high frequency content of the oscillatory transient signal. This finding indicates that the voltage sag and oscillatory transient are low and high frequency signals, respectively. This observation provides vital information for further analysis.

Feature extraction is performed by applying standard statistical techniques to the S-matrix contours and directly on the S-matrix. The following four features ( $F 1$ $F 4)$ extracted from the S-matrix are used as a basis for classification.

$F 1$ is the mean value of the amplitude versus time graph from the S-matrix near the fundamental frequency,

$$
F 1=\sum_{j=1}^{N}\left[\max _{i=15}^{19} S(i, j)-F 1\right] / N
$$

where $N$ is the number of rows in the S-matrix. Given that the sampling frequency is $3.2 \mathrm{kHz}$ and the fundamental frequency of the signals is $50 \mathrm{~Hz}$, the frequency deviation between the adjacent two rows is $3.125 \mathrm{~Hz}$. Hence, "near the fundamental frequency" is obtained from row 15 to 19 of the S-matrix.

$F 2$ is the standard deviation of the magnitude versus time spectrum obtained from the S-matrix near the fundamental frequency,
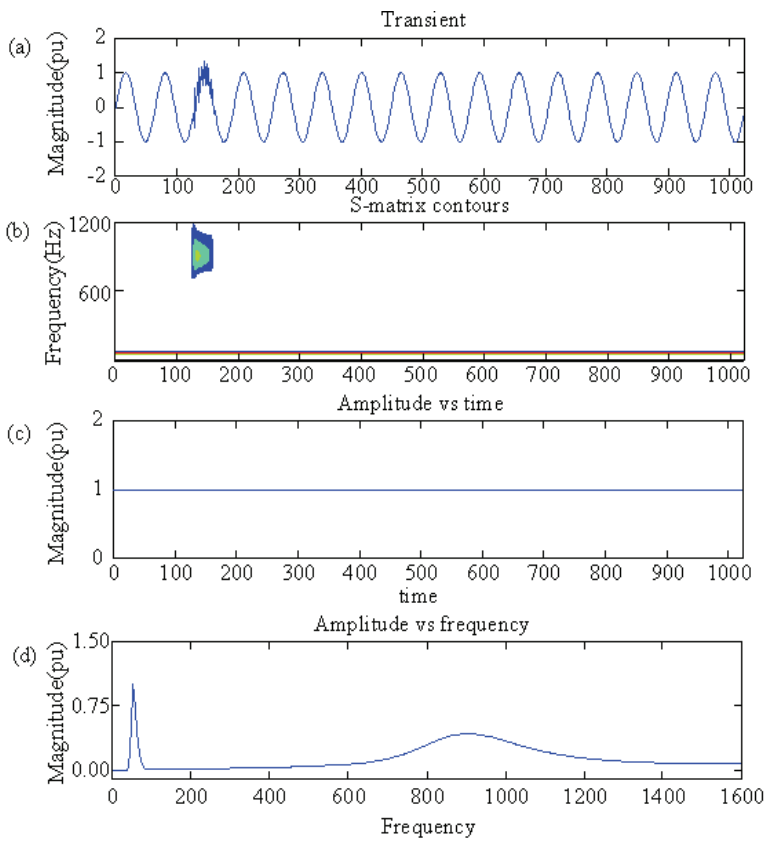

Fig. 3. (a) Transient waveform; (b) S-matrix contours of transient; (c) Magnitude-time curve from S-matrix; (d) Amplitude-frequency curve from S-matrix 


$$
F 2=\sqrt{\sum_{j=1}^{N}\left[\max _{i=15}^{19} S(i, j)-F 1\right]^{2} / N}
$$

F3 is the harmonic distortion of the S-matrix. The denominator of $F 3$ is the square root of the square sum of the peak near the fundamental frequency of the S-matrix (rows 12-33) for the normal sinusoidal signal, recorded as $V$. The numerator is the square root of the square sum of the signal to be classified, except the points near the peak of the fundamental frequency.

$$
F 3=\sqrt{\sum_{i=2}^{11}\left[\max _{j=1}^{N} S(i, j)\right]^{2}+\sum_{i=34}^{513}\left[\max _{j=1}^{N} S(i, j)\right]^{2}} / V
$$

With the same denominator of $F 3, F 4$ is the ratio between the square root of the square sum of harmonics above $300 \mathrm{~Hz}$ and $V$,

$$
F 4=\sqrt{\sum_{i=257}^{513}\left[\max _{j=1}^{N} S(i, j)\right]^{2}} / V
$$

After preliminary selection based on the essential characteristics of PQ signals and usually chosen features in application, the features for classification may need increasing, decreasing, or exchanging to guarantee the reliability of classification results. The four features in this paper are selected through the mentioned-above process until they are properly for classification of all patterns. The number of features proposed in this paper is less than most of the references under the same or similar condition. With the features being the inputs of a fuzzy classifier, fewer number of features generally indicates a simpler classifier structure (i.e., a simple fuzzy reasoning process and fewer fuzzy rules) and a smaller amount of calculation.

\section{Fuzzy Inference System}

The fuzzy sets and fuzzy rule base are the two key elements of a fuzzy inference system. A fuzzy set can be fully defined by its membership functions. A fuzzy rule base offers human-like reasoning capabilities and provides transparent inference mechanism, and each rule in fuzzy rule base must contain the antecedent part and consequent part. In the proposed method, four features extracted from ST for all signal patterns are fed to the fuzzy inference system with the improved Gaussian membership functions. A fuzzy rule base is then developed for exact classification of disturbance signals for 11 classes.

Specifically, based on the distribution of membership functions, certain features are selected from $F 1-F 4$ to represent the essential information of each pattern for recognition, and fuzzy rules are defined as one-to-one correspondence between the input fuzzy variables and the output classification results. Besides, the antecedent part of fuzzy rules is calculated and the result is generated as the degree of fulfillment (DOF) of the antecedent part. After reasoning process from the antecedent part to consequent part, the maximum membership degree method is used to select the pattern with the maximum DOF of all signal patterns. And the selected pattern is used as the final classification result.

\subsection{Membership function}

According to (4)-(7), the value of $F 1-F 4$ for all cases can be obtained from the S-matrix and the universe of each feature can be calculated. Thereafter, on the basis of the distribution of feature values, the membership functions corresponding to the pattern linguistic value in the respective universe can be defined. The membership functions can also determine whether the pattern of 11 disturbances can be identified by using these features.

The feature distribution range of all sample values for all patterns is the universe of this feature, and the feature distribution range of each pattern is the general range of membership function for this pattern. The following takes $F 1$ for example.

$F 1$ is one of the input variables of the fuzzy classifier, and its linguistic value is directly set as the pattern of PQ disturbances, i.e., normal, sag, swell, interruption, harmonics,

Table 2. Input variables of all membership functions

\begin{tabular}{c|c|c|c}
\hline Features & $F 1$ & $F 2$ & $F 3$ \\
\hline C1 & F1Normal & F2Normal & F3Normal \\
C2 & F1Sag & F2Sag & F3Sag F4Swell \\
C3 & F1Swell & F2Swell & F3Interruption \\
C4 & F1Interruption & F2Interruption & F4Swell \\
C5 & F1Harmonics & F2Harmonics & F3Flicker \\
C6 & F1Flicker & F2Flicker & F3Transient \\
C7 & F1Transient & F2Transient & F3Spike \\
C8 & F1Spike & F2Spike & F4Transient \\
C9 & F1Notch & F2Notch & F4Spike \\
C10 & F1sag_Harmonic & F2sag_Harmonic & F4Notch \\
C11 & F1swell_Harmonic & F2swell_Harmonic & F3swell_Harmonic \\
\hline
\end{tabular}


flicker, transient, spike, notch, sag with harmonic, and swell with harmonic, which are recorded as F1Normal, F1Sag, F1Swell, F1Interruption, F1Harmonics, F1Flicker, F1Transient, F1Spike, F1Notch, F1sag_Harmonic, and F1swell Harmonic, respectively. The definition of all membership functions of input variables are shown in Table 2.

Based on the requirements of disturbances classification, the membership functions with the flat-topped distribution are selected to ensure the aggregation degree of membership function for each pattern. The improved Gaussian membership function, which is simpler than other flattopped functions likely trapezoid and mountain shaped function, is used for classification. The improved Gaussian membership function is defined as follows:

$$
G(x)= \begin{cases}e^{-\left(\frac{x-a}{\sigma}\right)^{2}}, & x \leq a \\ 1, & a<x \leq b \\ e^{-\left(\frac{x-b}{\sigma}\right)^{2}}, & x>b\end{cases}
$$

where $a, b$, and $\sigma$ are the shape parameters of membership function

$$
\begin{aligned}
a & =F 1_{\text {min }}+0.1\left(F 1_{\text {max }}-F 1_{\text {min }}\right) \\
b & =F 1_{\text {min }}+0.9\left(F 1_{\text {max }}-F 1_{\text {min }}\right) \\
& =F 1_{\text {max }}-0.1\left(F 1_{\text {max }}-F 1_{\text {min }}\right) \\
\sigma & =0.19\left(F 1_{\text {max }}-F 1_{\text {min }}\right)
\end{aligned}
$$

where $F 1_{\min }$ and $F 1_{\max }$ are the minimum and maximum of this pattern of signals, respectively. In this case, the area of membership degree with 1 covers the $80 \%$ range from $F 1_{\text {min }}$ to $F 1_{\text {max }}$, and the membership degree is approximately 0.75 with $F 1=F 1_{\max }$ or $F 1_{\text {min }}$.

All cases of $F 1$ in the simulation are shown as Fig. 4, wherein the abscissa is the value of $F 1$. To distinguish $F 1$ from the different PQ disturbances, the ordinate in Fig. 4 is defined as $\mathrm{C} 1-\mathrm{C} 11$, which corresponds to normal, sag,

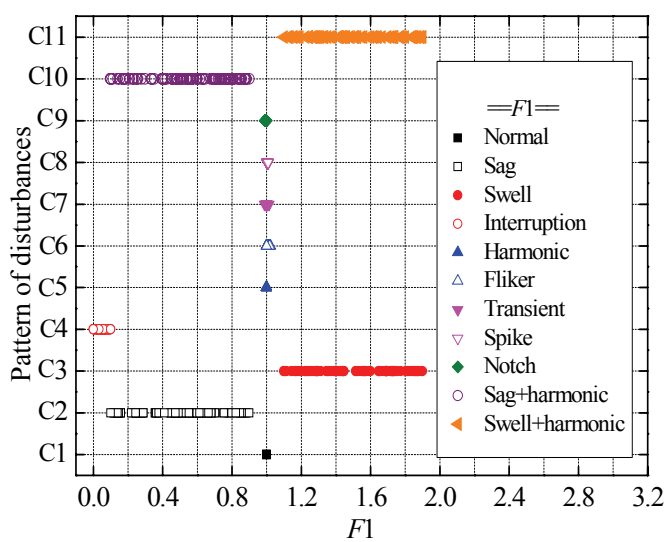

Fig. 4. Distribution of $F 1$. swell, interruption, harmonics, flicker, transient, spike, notch, sag with harmonic, and swell with harmonic from bottom to top.

The universe of $F 1$ is $[0,2]$ and is obtained by rounding down the minimum and rounding up the maximum of the variable range of $F 1$ [5.40489E-4, 1.90017] in Fig. 4. As abovementioned, the number of linguistic values in its universe is 11 , corresponding to the values of $F 1$ for all patterns of disturbances. Therefore, depending on the minimum and maximum features of each pattern, the membership function of this linguistic value can be calculated by (9). For example, the minimum and maximum features of sag (shown as the ordinate value 1 in Fig. 4) are respectively 0.09971 and 0.90027 plugged into (9), the membership function of F1Sag is obtained as $a=0.179766$, $b=0.820214$, and $\sigma=0.1521064$. By analogy, the membership functions of all linguistic values for $F 1$ are obtained (Fig. 5).

The membership functions of all linguistic values for F2-F4 can also be obtained (Fig. 6 - 8). By contrasting Fig. 5 to Fig. 8, the distribution of membership function among different features is clearly different; thus, the distinct characteristics among different patterns of disturbance are

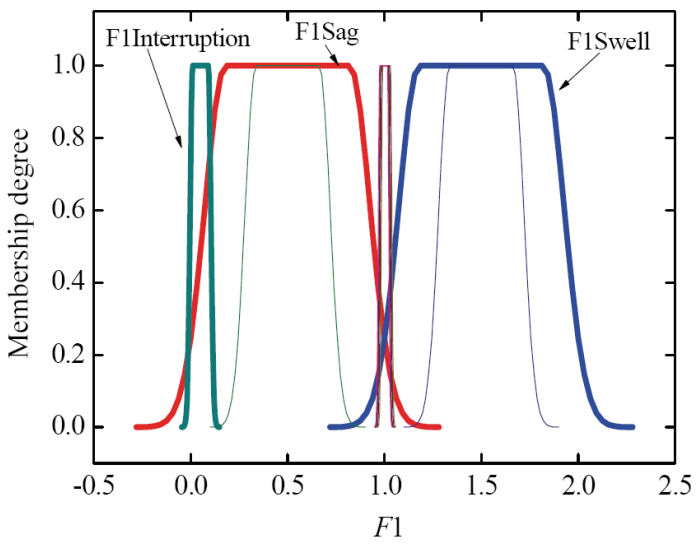

Fig. 5. Membership functions for $F 1$. Here, only the three patterns distinguished simply by $F 1$ are marked for clarity.

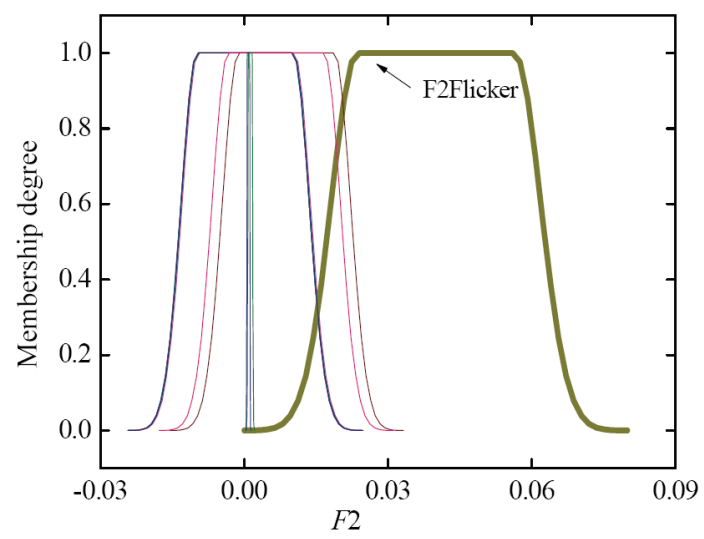

Fig. 6. Membership functions for $F 2$. Here, only the one pattern distinguished simply by $F 2$ is marked for clarity. 


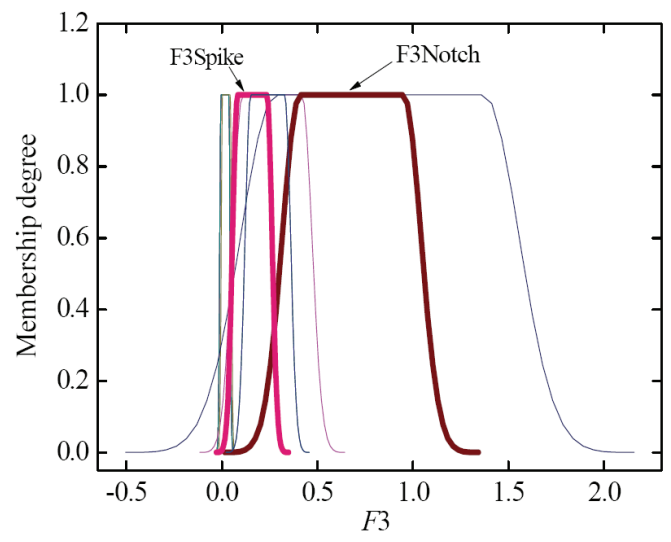

Fig. 7. Membership functions for $F 3$. Here, only the two patterns distinguished simply by $F 3$ are marked for clarity.

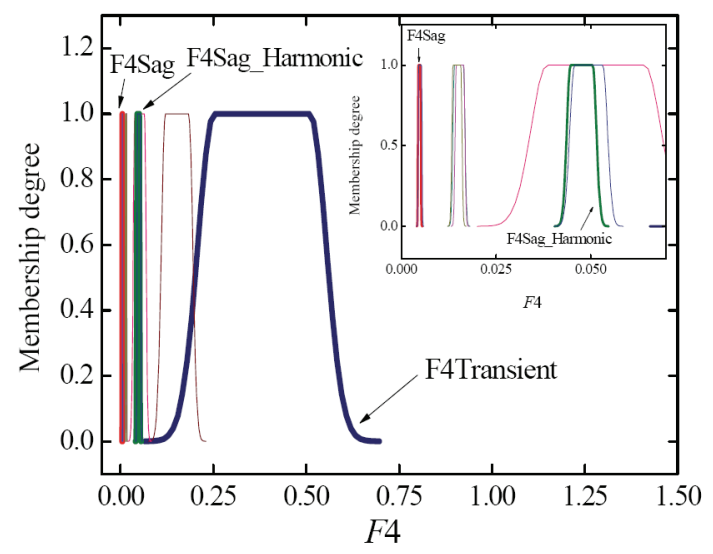

Fig. 8. Membership functions for $F 4$. Here, only the two patterns distinguished simply by $F 4$ are marked for clarity.

clearly reflected.

\subsection{Fuzzy rules}

After defining the membership functions for the various fuzzy sets of the extracted features from the S-matrix, a fuzzy rule base is formed for the recognition of disturbances. For the automatic recognition of 11 classes of disturbance patterns, the number of fuzzy rules is equal to the number of disturbance classes because these disturbance classes are used to define the linguistic values of fuzzy input variables.

In Fig. 5, the values of $F 1$ for sag, swell, interruption, sag with harmonic, and swell with harmonic have significant differences from the other six types of PQ disturbances. Furthermore, interruption can be merely recognized depending on $F 1$. The values of sag and sag with harmonic are clearly distinct from swell and swell with harmonic. However, the group of two similar signals is superimposed on each other (Fig. 8) and needs F4 to be classified. Thus, the aforementioned five patterns of classified signals can be identified by using $F 1$ and $F 4$. Others require more features to be identified because the value distribution of $F 1$ focused on the narrow area near one.

Fig. 6 shows that the values of flicker are different from normal, harmonics, spike, and notch such that flicker can be recognized by $F 2$. Moreover, the values of normal and harmonics are clearly distinct from spike and notch. However, the group of two similar signals is superimposed on each other and needs $F 3$ to be classified. Fig. 7 shows the values of normal and harmonics, with spike and notch obviously distinguished, respectively. Thus, the combination of $F 2$ and $F 3$ is used to classify the aforementioned four patterns of disturbances. The transient can be recognized depending on F4 (Fig. 8).

On the basis of the proposed analysis, the following fuzzy if-then rules are used to build the fuzzy rule base. Each if-then rule contains two parts: the antecedent part and consequent part. If the signal data for classification satisfy the fuzzy sets in the antecedent part, the signal data also satisfy the output class in the consequent part.

\section{Fuzzy rule base:}

Rule-1 if [ $(F 1$ is F1Normal) and (F2 is F2Normal) and ( $F 3$ is F3Normal)] then Signal is Normal

Rule-2 if ( $F 1$ is F1Sag) then Signal is Sag

Rule-3 if ( $F 1$ is F1Swell) then Signal is Swell

Rule-4 if ( $F 1$ is F1Interruption) then Signal is Interruption

Rule-5 if [ $F 1$ is F1Harmonics) and (F2 is F2Harmonics) and ( $F 3$ is $\mathrm{F} 3$ Harmonics) and ( $F 4$ is not F4Harmonics)] then Signal is Harmonics

Rule-6 if [(F1 is F1Flicker) and (F2 is F2Flicker) and (F3 is F3Flicker)] then Signal is Flicker

Rule-7 if [(F1 is F1 Transient) and (F2 is F2 Transient) and (F3 is F3Transient) and (F4 is F4Transient)] then Signal is Transient

Rule- 8 if [(F2 is F2Spike) and (F3 is F3Spike) and (F4 is F4Spike)] then Signal is Spike

Rule-9 if [(F2 is F2Notch) and (F3 is F3Notch) and (F4 is F4Notch)] then Signal is Notch

Rule-10 if ( $F 1$ is F1Sag_Harmonic) and ( $F 4$ is $\mathrm{F} 4$ Sag_Harmonic) then Signal is Sag_Harmonic

Rule-11 if ( $F 1$ is F1Swell_Harmonic) and ( $F 4$ is F4 Swell Harmonic) then Signal is Swell Harmonic

"Not" is the logical "negation" operation in Rule-5.

In a rule, DOF of the antecedent part is calculated to implicate the antecedent part and consequent part, shown in (10). The output of rule is the class of PQ signals corresponding with the consequent part.

Rule-1 DOF1=min(F1Normal, F2Normal, F3Normal)

Rule-2 DOF2=F1Sag

Rule-3 DOF3=F1Swell

Rule-4 DOF4=F1Interruption

Rule-5 DOF5=min(F1Harmonics, F2Harmonics, F3Harmonics, (1-F4Harmonics))

Rule-6 DOF6=min ( F1Flicker, F2Flicker, F3Flicker) (10)

Rule-7 DOF7=min(F1Transient, F2Transient, F3Transient, 
Table 3. DOF of the antecedent part using random data in fuzzy rule base $(\mathrm{SNR}=20 \mathrm{~dB})$

\begin{tabular}{c|c|c|c|c|c|c|c|c|c|c|c}
\hline Patterns & DOF1 & DOF2 & DOF3 & DOF4 & DOF5 & DOF6 & DOF7 & DOF8 & DOF9 & DOF10 & DOF11 \\
\hline C1 & 1 & 0.245 & 0.245 & 0 & 0.202 & $8.4 \times 10^{-4}$ & 0 & $7.9 \times 10^{-5}$ & 0.007 & 0 & 0 \\
C2 & 0 & 0.896 & 0.232 & 0.445 & 0 & 0 & 0 & 0 & 0 & 0.843 & $1 \times 10^{-16}$ \\
C3 & 0 & 0.207 & 0.899 & 0 & 0 & 0 & 0 & 0 & 0 & $1.4 \times 10^{-15}$ & 0.787 \\
C4 & 0 & 0.248 & 0.178 & 0.755 & 0 & 0 & 0 & 0 & 0 & 0 & $4.6 \times 10^{-9}$ \\
C5 & 0 & 0.245 & 0.246 & 0 & 0.755 & 0 & 0 & $9.2 \times 10^{-5}$ & $1.4 \times 10^{-16}$ & $3.9 \times 10^{-4}$ & $5.9 \times 10^{-27}$ \\
C6 & 0 & 0.246 & 0.244 & 0 & 0 & 1 & 0 & $9.2 \times 10^{-31}$ & $3.8 \times 10^{-31}$ & $8.7 \times 10^{-8}$ & $2.3 \times 10^{-8}$ \\
C7 & 0 & 0.245 & 0.245 & 0 & $2.8 \times 10^{-6}$ & 0 & 0.767 & $5.8 \times 10^{-5}$ & 0 & $2.6 \times 10^{-5}$ & $1.3 \times 10^{-14}$ \\
C8 & 0 & 0.213 & 0.280 & 0 & 0 & 0 & $1.6 \times 10-4$ & 0.758 & $4.7 \times 10^{-7}$ & $1.5 \times 10^{-4}$ & $2.1 \times 10^{-3}$ \\
C9 & 0 & 0.276 & 0.217 & 0 & 0 & $6.4 \times 10^{-24}$ & $2.8 \times 10-5$ & $4.9 \times 10^{-3}$ & 0.778 & 0.009 & 0.021 \\
C10 & 0 & 0.759 & 0.261 & 0 & 0 & 0 & 0 & 0 & 0 & 0.997 & $6.7 \times 10^{-8}$ \\
C11 & 0 & 0.256 & 0.761 & 0 & 0 & 0 & 0 & 0 & 0 & $2.7 \times 10^{-13}$ & 0.851 \\
\hline
\end{tabular}

F4Transient)

Rule-8 DOF8=min(F2Spike, F3Spike, F4Spike)

Rule-9 DOF9=min(F2Notch, F3Notch, F4Notch)

Rule-10 DOF10 $=\min ($ F1Sag_Harmonic, F4Sag_Harmonic)

Rule-11 DOF11=min(F1Swell_Harmonic,

F4Swell_Harmonic)

To obtain the final output result of fuzzy rule base, the maximum membership degree approach is used to aggregate the consequents across all the rules. The output class of disturbance patterns using the proposed fuzzy recognition system is also provided.

\section{Results and Discussion}

\subsection{Simulation results}

To illustrate the feasibility of the proposed method, 100 different cases of disturbances for each pattern are utilized. Furthermore, to have a better illustration of the robustness of the proposed method and to achieve the practical condition, signal cases are mixed with different Gaussian noise levels. Each example has signal-to-noise ratio (SNR) values of 20 and $50 \mathrm{~dB}$. As an example, Table 3 shows the DOF of the antecedent part by using a random set of cases in rule base. Fig. 9 shows the classification accuracy of the proposed method for simulation.

Results show that this method has high robustness and classification accuracy. The rule base and computation process in this method are simplified compared with the traditional fuzzy classification.

\subsection{Results of measured data}

To further evaluate the effectiveness of the proposed method, it is checked by some actual PQ disturbances. These disturbances are obtained from a PQ waveform library, including a number of common signature waveforms for various power system faults and PQ events. The waveforms in this library are available for download in the IEEE

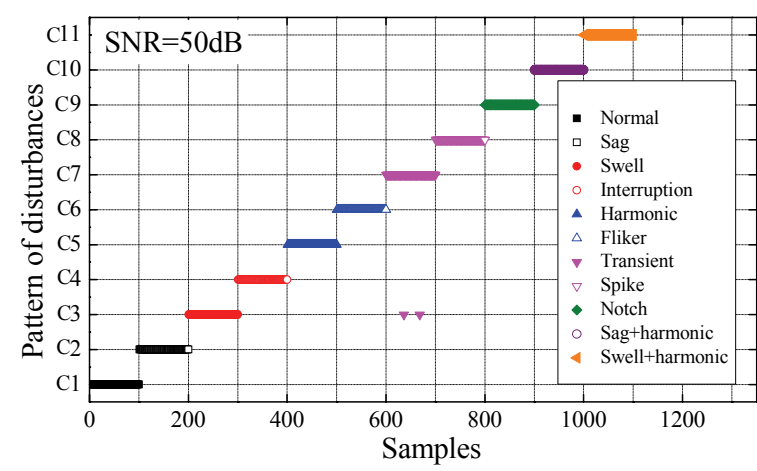

(a) Overall accuracy is $99.82 \%$

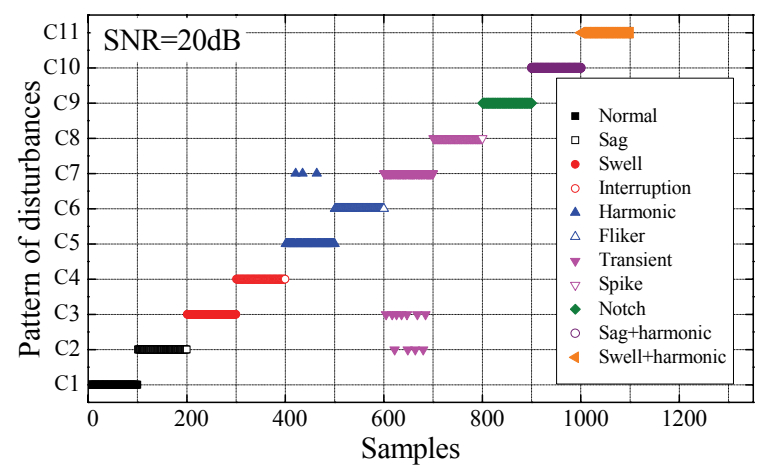

(b) Overall accuracy is $98.72 \%$

Fig. 9. Classification accuracies for simulation

PQDIF (IEEE Std. 1159.3) format. A total of 20 cases of each type are extracted to be used as the inputs of the proposed method.

Fig. 10 shows representative waveform of the measured data, whereas Fig. 11 shows the classification accuracy of the proposed method for measured data. Results show that this method has proper accuracy and can be applied.

\subsection{Performance comparison and discussion}

The performance of the proposed method and other literature methods are compared in Table 4. As seen in this table, the performance results of this method are desirable 
Table 4. Performance comparison with different methods

\begin{tabular}{|c|c|c|c|c|c|}
\hline \multirow[b]{2}{*}{ Reference } & \multirow[b]{2}{*}{ Method } & \multirow{2}{*}{$\begin{array}{l}\text { Feature } \\
\text { number }\end{array}$} & \multirow[b]{2}{*}{ Investigated PQ disturbance type } & \multicolumn{2}{|c|}{ Overall Accuracy (\%) } \\
\hline & & & & $\begin{array}{c}\text { Simulation } \\
(\mathrm{SNR}=20 \mathrm{~dB})\end{array}$ & $\begin{array}{c}\text { Measured } \\
\text { data }\end{array}$ \\
\hline$[4]$ & $\mathrm{SSD}+\mathrm{DT}$ & 6 & $\begin{array}{l}\text { Normal, sag, swell, interruption, harmonics, transient, } \\
\text { flicker, notch, sag + harmonic, spike, swell + harmonic }\end{array}$ & 96.7 & - \\
\hline$[6]$ & $\mathrm{WT}+\mathrm{SVM}$ & 16 & $\begin{array}{l}\text { Sag, swell, interruption, harmonics, flicker, transients, } \\
\text { sag + harmonic, swell + harmonic }\end{array}$ & 97.1 & 98.3 \\
\hline [9] & WT + HMM & 6 & Normal, sag, swell, harmonics, flicker, transients, & 98.0 & - \\
\hline [10] & $\mathrm{ST}+\mathrm{PNN}$ & 4 & $\begin{array}{l}\text { Normal, sag, swell, interruption, harmonics, transient, } \\
\text { flicker, notch, sag + harmonic, spike, swell + harmonic }\end{array}$ & 93.2 & - \\
\hline [14] & $\begin{array}{l}\text { WT + fuzzy } k \text {-nearest } \\
\text { neighbour }\end{array}$ & 16 & $\begin{array}{l}\text { Sag, swell, interruption, harmonics, transient, flicker, notch, } \\
\text { sag + harmonic, spike, swell + harmonic }\end{array}$ & 89.1 & 96.3 \\
\hline$[15]$ & $\mathrm{ST}+\mathrm{DT}-\mathrm{F}$ & 6 & $\begin{array}{l}\text { Sag, swell, interruption, harmonics, notch, flicker, transients, } \\
\text { Sag + harmonic, spike, swell + harmonic }\end{array}$ & 95.8 & 95.2 \\
\hline$[22]$ & $\begin{array}{l}\text { ST + fuzzy expert } \\
\text { systems with CF }\end{array}$ & 5 & $\begin{array}{l}\text { Normal, sag, swell, interruption, harmonics, notch+harmonics, } \\
\text { transient, flicker, sag + harmonic, spike + harmonics, swell + harmonic }\end{array}$ & 99.2 & - \\
\hline$[23]$ & WT(MRA) + FFT & 6 & $\begin{array}{l}\text { Normal, sag + noise, interruption, swell + noise harmonics, } \\
\text { harmonic+ noise sag, sag + harmonic, swell, swell + harmonic }\end{array}$ & 99.0 & - \\
\hline $\begin{array}{l}\text { In this } \\
\text { article }\end{array}$ & $\begin{array}{l}\mathrm{ST}+\text { fuzzy using } \\
\text { pattern linguistic } \\
\text { values }\end{array}$ & 4 & $\begin{array}{l}\text { Normal, sag, swell, interruption, harmonics, transient, flicker, } \\
\text { notch, sag + harmonic, spike, swell + harmonic }\end{array}$ & 98.7 & 98.6 \\
\hline
\end{tabular}

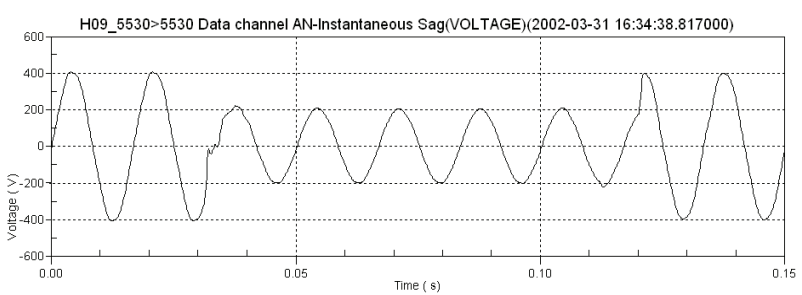

(a) Waveform of voltage sag

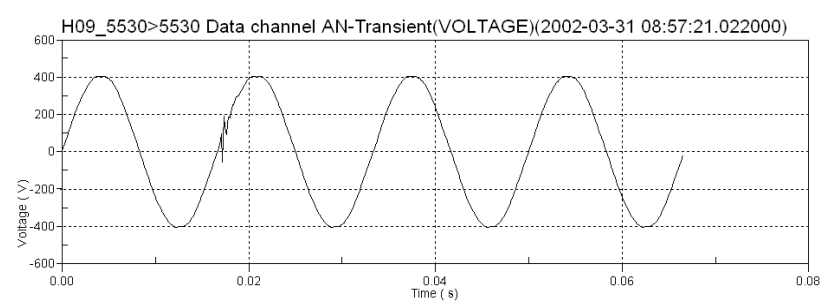

(b) Waveform of transient

Fig. 10. Representative measured data of PQ disturbances meet IEEE Std. 1159.3

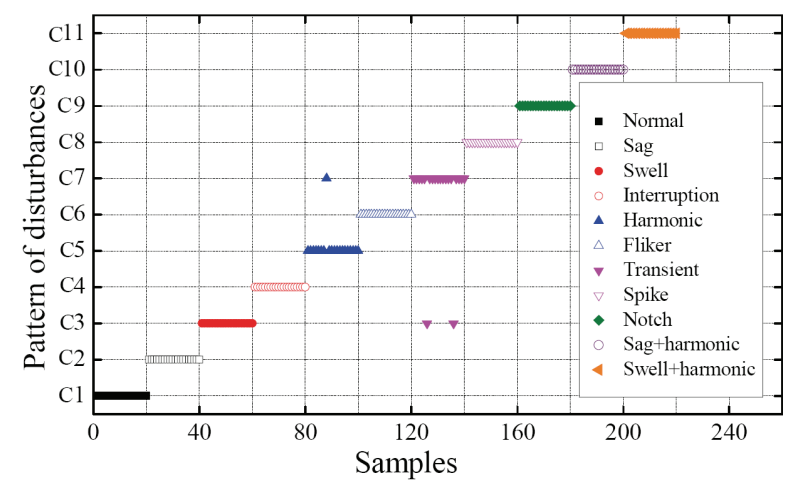

Fig. 11. Classification accuracies for measured data. The overall accuracy is $98.6 \%$. in contrast with the classification accuracies proposed in other works.

The advantage of the new fuzzy classification described in this article is the consideration of the combined disturbances and instantaneous disturbances, as some of them are not recognized in [8, 22]. The classification accuracy of $[8,22]$ is $98 \%$ and $99 \%$, respectively; whereas the classification accuracy of this study is $98.7 \%$ for the simulation. Although the classification accuracy in [22] is slightly better, the partial results are obtained without noise; differently, the results in this study are obtained under the condition of noise level. Furthermore, the classification of disturbances is completed only by four features by using the proposed algorithm, which is the minimum of the feature numbers in Table 4.

To evaluate the simulation performance between the proposed method and other recognition methods, a comparison among [4, 6, 10, 15] is performed. The classification accuracy of $[4,6,10,15]$ is $96.7,97.1,93.2$, and $95.8 \%$, respectively. Besides, the classification accuracy of this study and the mentioned investigations are mixed with the noise value of $20 \mathrm{~dB}$.

A comparison of the simulation results is made between the results of this article and the results of [14, 22] by using traditional fuzzy classification. The classification accuracy of [14] is $89.1 \%$ under the same noise condition, which is lower than that of this study. In [22], the reported results have high accuracy, but the accuracy increased up to $99.2 \%$ with the use of adaptive particle swarm optimization technique combined with fuzzy logic for optimizing membership functions of the concerned fuzzy sets. The proposed method without using optimization algorithms achieves proper classification accuracy, thus ensuring the effectiveness of recognition and reducing the difficulty of classification algorithm.

Finally, to evaluate the practical performance of the 
proposed algorithm, a comparison among each method in Table 4 is performed, except for [4, 9, 10, 22, 23], by using measured data. The classification accuracy of $[6,14,15]$ for measured data is $98.3,96.3$, and $95.2 \%$, respectively, whereas the classification accuracy of this study is $98.6 \%$. Thus, the proposed method has proper accuracy and passes the test with high accuracy for measured data.

\section{Conclusion}

In this paper, an attempt has been made to identify and classify PQ disturbances by using ST and classifier based on pattern linguistic value, which utilizes the internal relations between the parameters and rule base of the classifier. The design process of linguistic value numbers and membership function parameters does not only relate to the values of input variables but also to the classification purpose directly. Given that the number of linguistic values is equal to the number of disturbance patterns, the parameters of membership function are directly calculated from the relevant data of each pattern. Furthermore, the number of fuzzy rules is the same as the number of disturbance patterns and can be directly obtained from the analysis of the distribution of input values.

The performance results for simulation and measured data show that the proposed method has the ability of recognizing and classifying different PQ disturbances efficiently even with insufficient and noisy data. The number of features used in this method is reduced to four, which is significantly less than other similar works. This method also reduces the dimensionality and complexity of the classifier. Furthermore, considering the characteristics and parameters of disturbances, the complexity of the fuzzy reasoning process and rule base are substantially reduced compared with the traditional fuzzy inference system. Moreover, the calculation is reduced in this study to ensure the classification effectiveness of the classifier. This work provides a great potential method for applications, particularly under the condition of unfavorable hardware resources.

\section{References}

[1] F. Z. Zhao, R. G. Yang, "Voltage sag disturbance detection based on short-time Fourier transform," Proceedings of the CSEE, vol. 27, no. 10, pp. 28-34, 109, Apr. 2007.

[2] T. X. Zhu, S. K. Tso, and K. L. Lo, "Wavelet-based fuzzy reasoning approach to PQ disturbance recognition," IEEE Trans. Power Deliv., vol. 19, no. 4, pp. 1928-1935, Oct. 2004.

[3] R. G. Stockwell, L. Mansinha, and R. P. Lowe, "Localization of the complex spectrum: the S-trans- form," IEEE Transactions on Signal Processing, vol. 44, no. 4, pp. 998-1001, Apr. 1996.

[4] M. S. Manikandan, S. R. Samantaray, I. Kamwa, et.al, "Detection and classification of power quality disturbances using sparse signal decomposition on hybrid dictionaries," IEEE Transactions on Instrumentation and Measurement, vol. 64, no. 1, pp. 594605, Jan. 2015.

[5] S. Kalyani and K.S. Swarup, "Classification and assessment of power system security using multiclass SVM," IEEE Trans. Syst. Man and Cybernetics Part C-Applications and Reviews, vol. 41, no. 5, pp. 753758, Sep. 2011.

[6] K. Manimala, K. Selvi, and R. Ahila, "Optimization techniques for improving power quality data mining using wavelet packet based support vector machine," Neurocomputing, vol. 77, no. 1, pp. 36-47, Feb. 2012.

[7] H. Eristi and Y. Demir, "Automatic classification of power quality events and disturbances using wavelet transform and support vector machines," IET Gener. Transm. Distrib., vol. 6, no. 10, pp. 986-976, 2012.

[8] N. Thai and L. Yuan, "Power quality disturbance classification utilizing S-transform and binary feature matrix method," Electric Power Systems Research, vol. 79, pp. 569-575, Apr. 2012.

[9] T. K. Abdel-Galil, E. F. El-saadany, and A. M. Youssef, et.al, "Disturbance classification using hidden Markov models and vector quantization," IEEE Trans. Power Deliv., vol. 20, no. 3, pp. 2129-2135, Jul. 2005.

[10] S. Mishra, C. N. Bhende, and B. K. Panigrahi, "Detection and classification of power quality disturbances using S-transform and probabilistic neural network," IEEE Trans. Power Deliv., vol. 23, no. 1, pp. 280-287, Jan. 2008.

[11] O. Mário, V. C. Denis, and D. F. Odilon, et.al, "Power quality analysis applying a hybrid methodology with wavelet transforms and neural networks," Electrical Power and Energy Systems, vol. 31, no.5, pp. 206212, Jun. 2009.

[12] K. Raj, S. Bhim, and T. S. Dilip, "Recognition of single-stage and multiple power quality events using Hilbert-Huang transform and probabilistic neural network," Electrical Power and Energy Systems, vol. 43, no. 6, pp. 607-619, Apr. 2015.

[13] A. N. Chirag and K. Prasanta, "Power quality disturbance classification employing S-transform and three-module artificial neural network," Int. Trans. Electr. Energ. Syst. vol. 24, no. 9, pp. 1301-1322, Sep. 2014.

[14] B.K. Panigrahi and V.R. Pandi, "Optimal feature selection for classification of power quality disturbances using wavelet packet-based fuzzy k-nearset neighbour algorithm," IET Gener. Transm. Distrib., vol. 3, no. 3, pp. 296-306, Mar. 2009.

[15] S. R. Samantaray, "Decision tree-initialised fuzzy rule-based approach for power quality events classi- 
fication," IET Gener. Transm. Distrib., vol. 4, no. 4, pp. 538-551, Apr. 2010.

[16] A. A. Abdelsalam, A. A. Eldesouky, and A. A. Sallam, "Classification of power system disturbances using linear Kalman filter and fuzzy-expert system," Int. Jour. Elec. Power, vol. 43, no. 1, pp. 688-695, Dec. 2012.

[17] A. Rodriguez, J. A. Aguado, and F. Martin, "Rulebased classification of power quality disturbances using S-transform,” Electric Power Systems Research, vol. 86, pp. 113-121, May 2012.

[18] D. Granados-Lieberman, R.J. Romero-Troncoso, and R.A. Osornio-Rios, et.al, "Techniques and methodologies for power quality analysis and disturbances classification in power systems: a review," IET Gener. Transm. Distrib., vol. 5, no. 4, pp. 519-529, Apr. 2011.

[19] K. Rajiv and G. Rashmi, "Fuzzy lattice based technique for classification of power quality disturbances," Eur Trans. Electr. Power, vol. 22, no. 8, pp. 1053-1064, Nov. 2012.

[20] K. S. Yap, C. P. Lim, and M. T. Au, "Improved GART neural network model for pattern classification and rule extraction with application to power systems," IEEE Trans. Neural Networ., vol.22, no.12, pp. 23102323, Dec. 2011.

[21] S. K. Meher and A. K. Pradhan, "Fuzzy classifiers for power quality events analysis," Electr. Pow. Syst. Res., vol. 80, no. 1, pp. 71-76, Jan. 2010.

[22] H.S. Behera, P.K. Dashb, and B. Biswal, "Power quality time series data mining using S-transform and fuzzy expert system," Appl. Soft Comput., vol. 10, no. 3, pp . 945-955, Jun. 2010.

[23] S. A. Deokar, L. M. Waghmare, "Integrated DWTFFT approach for detection and classification of power quality disturbances," Electrical Power and Energy Systems, vol. 61, pp. 594-605, Oct. 2014.

[24] M. G. Ameen, E. Nesimi, L. S. Wen, "Automatic classification and characterization of power quality events," IEEE Trans. Power Deliv., vol. 23, no. 4, pp. 2417-2425, Oct. 2008.

[25] M. Uyar, S. Yildirim, M. T. Gencoglu, “An expert system based on s-transform and neural network for automatic classification of power quality disturbances," Expert Syst. Appl., vol. 36, no. 3, pp. 59625975, Apr. 2009.

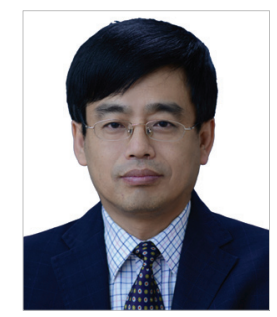

XiaoSheng Liu He received his B.S., M.S., and Ph.D. degrees from Harbin Institute of Technology (HIT), Harbin, China, in 1988, 1993, and 1999, respectively. He is now a Professor of HIT. His special fields of interest cover power quality analysis, active distribution network, and power line com-

munication.

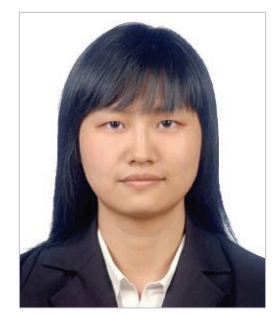

Bo Liu She received the B.S. and M.S. degrees in electrical engineering from Henan University of Science and Technology, Louyang, China, in 2007 and 2011, respectively. She is now pursuing her Ph.D. degree from the Department of Electrical Engineering, HIT. Her research interests include power quality analysis and control technology.

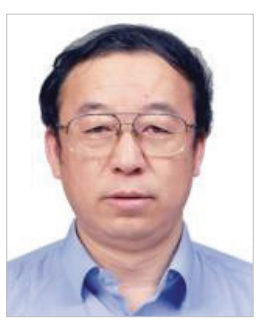

DianGuo Xu He received his B.S., M.S., and Ph.D. degrees from HIT, Harbin, China, in 1982, 1984, and 1989, respectively. He has been a Professor of HIT since 1994, and is also currently the Vice President. His research interests include renewable energy generation technology, multiterminal high-voltage direct current system based on voltage-source converters, power quality mitigation, and speed-sensorless vector-controlled motor drives. 